\title{
Benefícios da atividade física na prevenção e tratamento da obesidade: Uma breve
}

\section{revisão}

\section{Benefits of physical activity in the prevention and treatment of obesity: A brief review}

Beneficios de la actividad física en la prevención y el tratamiento de la obesidad: Una breve revisión

Taviane Pereira da Silva ORCID: https://orcid.org/0000-0002-1955-0331 Faculdade de Ensino Regional Alternativa, Brasil E-mail: flavianepereira1990@gmail.com

João Lenon Lima ORCID: https://orcid.org/0000-0002-2542-4657 Faculdade de Ensino Regional Alternativa, Brasi E-mail: jhclenon@gmail.com

Carlos Eduardo Lima Rocha de Oliveira ORCID: https://orcid.org/0000-0002-6072-1390 Faculdade Pitágoras, Brasil E-mail: eduardorocha_tc@hotmail.com Ralmony de Alcantara Santos ORCID: https://orcid.org/0000-0001-6211-3180 Centro Universitário CESMAC, Brasil E-mail: ralmonydealcantara@gmail.com

\begin{abstract}
Resumo
O objetivo do presente trabalho é avaliar os benefícios da atividade física na prevenção e tratamento da obesidade. Foram realizadas pesquisas apenas nacionais pelo, Google acadêmico, LILACS e Scielo com os seguintes anos de publicação de 2006 a 2018 por meio das palavras atividade física, exercício físico ou treinamento físico, exercício aeróbio, exercício resistido ou treinamento com pesos e obesidade pesquisados de maneira individual e em conjunto como obesidade, que proporcionaram rastrear artigos e monografias em formatos de pdf, que foram verificados pelo método de revisão de literatura. Diante de todos os estudos apresentados o que se pode observar é que a prática de atividade física promove a melhora na redução de gordura corporal através dos treinamentos aeróbio ou resistido por meio de exercícios como: caminhada, corrida e ciclismo, treinamento com pesos entre outros. Portanto, a pesquisa realizada por meio do método de revisão de literatura sobre atividade física e obesidade concluímos que a maioria dos artigos e monografias que foram analisados apresentaram efeitos benéficos da atividade física em indivíduos obesos. Pois a literatura apresenta que além da atividade física auxiliar na redução de gordura corporal também ajuda a diminuir os riscos de doenças associadas a ela.
\end{abstract}

Palavras-chave: Obesidade; Atividade física; Prevenção e tratamento.

\begin{abstract}
The objective of the present work is to evaluate the benefits of physical activity in the prevention and treatment of obesity. Only national searches were carried out by, academic Google, LILACS and Scielo with the next years of publication from 2006 to 2018 using the words physical activity, physical exercise or physical training, aerobic exercise, resistance exercise or weight training and obesity researched individually and jointly as obesity, which provide for tracking articles and monographs in pdf formats, which were verified by the literature review method. In view of all the studies generated, what can be observed is that the practice of physical activity promotes an improvement in the reduction of body fat through aerobic or resistance training through exercises such as: walking, running, and cycling, weight training, among others. Therefore, a survey conducted through the method of literature review on physical activity and obesity concluded that most articles and monographs that have been affected beneficial effects of physical activity in obese people. Because the literature shows that in addition to physical activity to help reduce body fat it also helps to reduce the risks of diseases associated with it.
\end{abstract}

Keywords: Obesity; Physical activity; Prevention and treatment.

\section{Resumen}

El objetivo del presente trabajo es evaluar los beneficios de la actividad física en la prevención y tratamiento de la obesidad. Solo se realizaron búsquedas nacionales por parte del académico de Google, LILACS y Scielo con los próximos años de publicación de 2006 a 2018 utilizando las palabras actividad física, ejercicio físico o entrenamiento 
físico, ejercicio aeróbico, ejercicio de resistencia o entrenamiento con pesas y obesidad investigadas de forma individual y conjunta. como la obesidad, que prevén el seguimiento de artículos y monografías en formato pdf, que fueron verificados por el método de revisión de literatura. A la vista de todos los estudios generados, lo que se puede observar es que la práctica de actividad física promueve una mejora en la reducción de la grasa corporal a través del entrenamiento aeróbico o de resistencia a través de ejercicios como: caminar, correr y andar en bicicleta, entrenamiento con pesas, entre otros. Por tanto, una encuesta realizada utilizando el método de revisión de literatura sobre actividad física y obesidad concluyó que la mayoría de artículos y monografías han afectado los efectos beneficiosos de la actividad física en personas obesas. Porque la literatura muestra que además de la actividad física para ayudar a reducir la grasa corporal también ayuda a reducir los riesgos de enfermedades asociadas a ella.

Palabras clave: Obesidad; Actividad física; Prevención y tratamiento.

\section{Introdução}

Segundo a pesquisa Nacional de saúde em 2013 que contatou que 56,9\% (Instituto Brasileiro de Geografia e Estatística, 2015) da população brasileira aponta excesso de peso e a obesidade chega aproximadamente 30 milhões de adultos (Brasil, 2019). A obesidade pode ser explicada, de forma simples, como um depósito de gordura corporal que esta relacionada a riscos para a saúde, por ter uma relação com diversas complicações metabólicas (Organização Mundial de Saúde, 2004).

A OMS (2004) destaca dois pontos principais para as causas de sobrepeso e obesidade, em geral o aumento da ingestão de alimentos altamente calóricos que contem gordura saturada e açúcar, e também com a diminuição de atividade física (AF) (Oliveira, 2008). Além do consumo alimentar ser destacado como um dos principais fatores da causa de sobrepeso e obesidade, alguns autores destacam também as causas ambientais (Oliveira, 2008), tais como: baixo nível de atividade física e muito tempo gasto com televisão, vídeo games e computadores (Almeida, Nascimento \& Quaioti, 2002).

A obesidade no período de infância e capaz de causar um aumento da quantidade de células adiposas, provocando um aumento exagerado de peso e acúmulo de gordura. Assim contribuindo para que as consequências direcionem para vida adulta (Juca, 2013). Sendo que a obesidade na fase de adolescência e relacionada ao surgimento de problemas cardiovasculares, diabetes tipo 2, doenças psicológicas e ainda sendo prejudicial para a postura, provocando mudanças no aparelho psicomotor e ocasionando dificuldades socioeconômicas na fase adulta (Camarneiro, 2011).

Estudos mostram que o sobrepeso e obesidade estão associados aos progressos de doenças, como: Diabetes Melitos tipo 2, Dislipidemia, Doenças cardiovascular, Doenças do aparelho respiratório, Doenças do aparelho locomotor e doenças da hipertensão arterial (Souza, Silva, Cavalcante, Lima, Caliandra \& Souza, 2018; Gaban, 2013).

Segundo Caspersen, Powell e Christenson (1985), o termo AF esta relacionado a qualquer movimento que o corpo realiza através do sistema muscular esquelético, que resulta e em um maior gasto energético se comparado a taxa metabólica em repouso. No entanto, o exercício físico (EF) inserido no tema AF, por ser uma atividade de caráter planejado, repetitivo e intencional, com objetivo de manter, melhorar um ou mais componentes da aptidão física (Silva \& Costa, 2011).

A maneira de se viver em um padrão de vida de modo ativo com a prática de $\mathrm{EF}$, tem várias vantagens na saúde das pessoas sendo também importante no cuidado, domínio, tratamento ou correção (Matsudo \& Matsudo, 2001). Com o desempenho frequente e habitual de AF, temos resultados positivos e satisfatórios sendo por meio dos benefícios proporcionados pela AF que entre eles estão os benefícios biológicos ou fisiológicos, psicológicos e cognitivos (Ceschine, 2007).

Segundo a OMS (2004) determina que a realização de AF segue como ideal para adultos uma prática moderada de pelo menos $150 \mathrm{~min}$. semanais ou uma prática de AF vigorosa de $75 \mathrm{~min}$. semanal, que podem ser divididos no mínimo em 10 min. de duração, sem definição de frequência por semana (Dias, Montenegro \& Monteiro, 2017). Em comformidade com Glaner (2003), exercícios aeróbios relaciona-se á função cardiovascular e pulmonar e podem ser descritas pela resistência aeróbica elemento da aptidão física associada à saúde (Lima, Levy \& Luiz, 2014).

Embora, o treinamento de força (TF), a musculação, não usem como fonte principal o tecido adiposo no momento do 
exercício e dispõe do sistema mais intenso as vias energéticas ATP-PC e glicólise anaeróbia, ocorrem um alto emprego de lipídios entre uma série e outra dos exercícios, por conta das atividades aeróbias aumentada, com objetivo de restabelecer os sistemas anaeróbios depletados. Além disso, o metabolismo continua alto após a prática de AF por muitas horas, o que aumenta a oxidação de gordura (Farias \& Rodrigues, 2009).

O treinamento aeróbico apresenta-se como uma forma muito usada em programas de emagrecimento em meio de EF sendo mais eficiente para redução de massa corporal, particularmente no tecido adiposo. Isto é, acontece pela elevação do déficit energético proporcionado pelo exercício, tanto durante como depois do término dele. Porém, o aumento do consumo de oxigênio mesmo após o término do esforço é uma das causas positivas do treinamento aeróbico em redução à perda e/ou manutenção do peso corporal (Dias et al., 2014).

O TF vem sendo usado como um dos recursos para o tratamento da obesidade tendo uma conduta auxiliar (The American College of Sports Medicine, 2002), pois o gasto calórico proporcionado pelo EF se apresenta como importante fator na redução de gordura corporal, e na melhora dos níveis de aptidão física (Neves, Martins, Souza, \& Júnior, 2015). Desta forma, o objetivo do presente trabalho é avaliar os benefícios da AF na prevenção e tratamento da obesidade.

\section{Metodologia}

O Presente estudo trata-se de uma pesquisa descritiva e de uma abordagem qualitativa. Segundo Martins (1994), a pesquisa descritiva "tem como objetivo a descrição das características de determinada população ou fenômeno, bem como o estabelecimento de relações entre variáveis e fatos". É o método de pesquisa que observa, registra, analisa, descreve e correlaciona fatos e fenômenos sem manipulá-los.

Dessa maneira, o presente trabalho foi realizado por meio de uma pesquisa, forma online nos indexadores, como: Google Acadêmico, Biblioteca Científica Eletrônica do SCIELO e LILAC, onde foram analisados apenas artigos e monografias nacionais entre os seguintes anos 2006 a 2018 pelo método de revisão bibliográfica. Nesses indexadores foram possíveis encontrar artigos e monografias relacionados ao tema atividade física e doenças não transmissíveis no qual foram escolhidos os que estavam voltados para atividade física e obesidade.

Foi realizado a busca dos artigos, nas bases eletrônicas, por meio das palavras "atividade física”, "exercício físico ou treinamento físico", "exercício aeróbio", "Treinamento aeróbico", "treinamento de força ou treinamento com pesos" e “obesidade" pesquisados de maneira individual e em conjunto como obesidade. Desta busca foram encontrados 78 artigos que ao fim foram excluídos pelo título 13 artigos restando um total de 65 .

Na busca inicial 65 (sessenta e cinco) artigos foram encontrados, sendo que 25 (vinte e cinco) na base de dados SCIELO e 10 (dez) no LILACS e 30 (trinta) no Google Acadêmico. Porém, após a leitura dos resumos disponíveis, foram excluídos 40 (quarenta) publicações, do SCIELO 15 (quinze), do LILACS 10 (dez) e do Google Acadêmico 15 (quinze).

Depois do refinamento, os 25 (vinte e cinco) artigos incluídos nesta etapa passaram para a segunda etapa, no qual foi realizada a leitura dos artigos de forma completa. Em ambas as etapas foram utilizados os critérios de inclusão e exclusão, como também tinham que estar voltado a temática sobre Atividade física e obesidade. Por sua vez, a amostra final foi composta por 25 artigos. A seguir fluxograma (Figura 1) representativo da busca realizada dos artigos nas bases de dados SCIELO, LILACS e Google Acadêmico. 
Figura 1. Esquema representativo da busca de artigos.

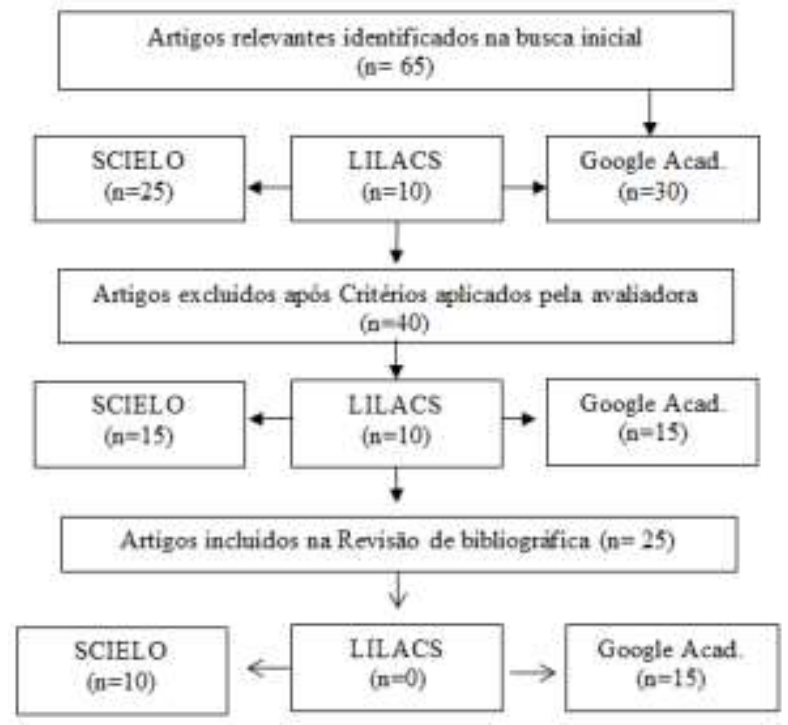

Fonte: Autores (2019).

\section{Revisão de literatura}

\subsection{Obesidade}

\subsubsection{Conceito}

A obesidade pode ser explicada, de forma simples, como um depósito de gordura corpo que esta relacionada a riscos para a saúde, por ter uma relação com muitas complicações metabólicas (OMS, 2004). Nas últimas décadas a obesidade teve um aumento principalmente nos países em desenvolvimento. Sua causa esta relacionada a diversos fatores genéticos, metabólicos, sociais, comportamentais e culturais. Além de ser destacada como um problema de saúde pública trazendo diversas consequências para a saúde afetando diretamente a qualidade de vida (Tavares, Nunes \& Santos, 2010).

A doença atrelada a má ingestão calórica que leva ao aumento de peso corporal. Porém, a obesidade se destaca com o aumento de peso excessivo. No entanto, temos como parâmetro para medir os níveis de obesidade na população e na prática clínica o Índice Massa Corporal (IMC), que é realizado através da relação entre o peso e estatura (Anjos, 2013). O que pode trazer riscos para saúde além do excesso de peso, a formar que esta gordura esta distribuída no corpo, na maioria das vezes se localizando na região abdominal, podendo trazer maiores riscos para a saúde. Desta forma, entre o grupo das doenças e agravos não transmissíveis (DANT) está obesidade (OMS, 2004).

\subsubsection{Dados sobre a obesidade}

A obesidade infantil vem aumentando muito nos últimos anos, com isso a maioria das crianças transformando-se em adultos obesos, tendo mais facilidade para adquirir várias doenças relacionadas à gordura corporal (Lima, 2012). A obesidade e o sobrepeso em crianças e adolescentes vêm aumentando, com isso estudos mostram a necessidade da avaliação do estado nutricional e de meios desenvolvidos pela OMS (2021) que indicam o IMC que desde o início de vida até os 19 anos. Em uma classificação que determina sobrepeso quando está entre os percentuais de IMC de 85 a 95 e obesidade quando esta acima de 95 do percentual do IMC (Lima, 2012).

Assim, os profissionais da área de saúde devem estar sempre atentos, quando uma criança ou adolescente, ultrapassar o nível do IMC acima de 85 do percentual (Lima, 2012). Dessa forma, Lima (2012) salienta que crianças com menos de oito anos que estão obesos, tendem a se tornarem adultos obesos com maiores chances de adquirir doenças na vida adulta. Com 
isso, tendo conseqüências como dificuldade de respiração, aumento do risco de fraturas, hipertensão arterial, problemas cardiovasculares e dentre outras. Enquanto, nas meninas o aumento está também relacionada a maiores riscos de problemas psicológicos e até a adquirir câncer de mama (Lima, 2012).

A obesidade infantil está se tornando uma grande preocupação. Segundo a pesquisa de orçamentos familiares (POF), 2008 - 2009 (IBGE, 2010) foram feitas no Brasil, para análise do estado nutricional de crianças, adolescentes e adultos. O acúmulo exagerado de peso em adolescentes modificou em ambos os sexos, de $16 \%$ a $19 \%$ nas regiões Norte e Nordeste e de $20 \%$ a $27 \%$ nas regiões Sudeste e Centro-Oeste. Em ambos os sexos o aumento de peso e mais continuo na área urbana, do que na zona rural (Lima, 2012).

A obesidade em crianças na faixa etária de 8 a 9 anos de idade vem aumentando muito nos últimos anos no país. Nos anos de 1989 a 2009, o número de meninos acima do peso duplicou, ultrapassando de 15\% para 34,8\%, logo a quantidade de obesos aumentou em mais de 300\% na mesma faixa etária de idade, passando de 4,1\% em 1989 para 16,6\% 2008- 2009 (Lima, 2012). Segundo a pesquisa Nacional de saúde em 2013 que contatou que 56,9\% (IBGE, 2015) da população brasileira aponta excesso de peso e a obesidade chega aproximadamente 30 milhões de adultos (Brasil, 2019).

De acordo com as pesquisas realizadas em 27 cidades, constatou que a frequência de excesso de peso foi de $52,3 \%$, com maior incidência entre os homens $(60,4 \%)$ se comparado às mulheres $(45,9 \%)$. Em relação à obesidade, a frequência de adultos obesos foi de 17,0\%, sendo maior nos homens do que nas mulheres (Brasil, 2019). Segundo a OMS, a obesidade pode ser classificada pelo IMC, sendo apontada como o acumulo de gordura anormal ou em excesso no organismo (Silva, 2017). A obesidade e definida de acordo com os distintos pontos de corte apresentados pela OMS, segundo Tabela 1 (Silva, 2017).

Tabela 1. Classificação do estado nutricional prevista pela OMS para adultos maiores de 18 anos.

\begin{tabular}{cc}
\hline IMC $\left(\mathrm{Kg} / \mathrm{m}^{2}\right)$ & Classificaçāo \\
\hline$<18,5$ & Baixo peso \\
$18,5-24,99$ & Normal - eutrófico \\
$25-29,99$ & Sobrepeso \\
$30-34,99$ & Obesidade grau I \\
$35-39,99$ & Obesidade grau II \\
$\geq 40$ & Obesidade grau III \\
\hline
\end{tabular}

Fonte: Silva (2017).

A obesidade abdominal é considerada um fator de risco livre para várias morbidades, por se destacar pelo aumento de tecido adiposo na região abdominal, que é apontado como obesidade abdominal ou visceral (Silva, 2017). A circunferência da cintura é uma medida de baixo custo e de fácil acesso para ser utilizada em estudos populacionais epidemiológico. Além disso, se mostra um excelente parâmetro de gordura visceral se comparado a outros indicadores antropométricos (Silva, 2017). A obesidade abdominal pode ser apontada conforme os seus pontos de corte determinados pela OMS: risco aumentado ou nível I e risco substancialmente aumentado ou nível II, segundo Tabela 2 (Silva, 2017).

Tabela 2. Pontos de corte utilizado pela OMS para classificação de obesidade abdominal.

\begin{tabular}{ccc}
\hline & Homens & Mulheres \\
\hline $\begin{array}{c}\text { Risco aumentado } \\
\text { Nivel I }\end{array}$ & $\geq 94 \mathrm{~cm}$ & $\geq 80 \mathrm{~cm}$ \\
$\begin{array}{c}\text { Risco substancialmente } \\
\text { aumentado/ Nivel II }\end{array}$ & $\geq 102 \mathrm{~cm}$ & $\geq 88 \mathrm{~cm}$ \\
\hline
\end{tabular}

Fonte: Silva (2017). 


\subsubsection{Causas de obesidade}

A obesidade é ocasionada pelo consumo exagerado de alimentos como; gordura, carboidratos e proteínas, assim, trazendo o aumento de gordura no organismo, por meio de alimentos que não são usados pelo organismo como fonte de energia (Oliveira, 2008). A obesidade é causada pelo consumo exagerado de alimento e menor gasto energético, sendo que para uma pessoa obesa o consumo de 9,3 Kcal é transformado em $1 \mathrm{~g}$ de gordura, por isso para uma pessoa se manter obesa só precisa ter um consumo igual ao gasto energético (Oliveira, 2008).

Além disso, o aumento das doenças relacionadas à obesidade retrata modificações na sociedade e nos comportamentos sociais como o crescimento econômico, modernização, urbanização e a praticidade para adquirir o alimento (Oliveira, 2008). Adicionalmente, Oliveira (2008) salienta que algumas pessoas têm maior facilidade para acumular gordura, e dificuldade para gastar energia, por causa do seu metabolismo. Portanto, o aumento de peso e por meio do maior consumo de calorias e menor nível de AF (Oliveira, 2008).

Por fim, Pinheiro, Freitas e Corso (2004), ressaltam um dos três fatores que causam a obesidade e a genética e os outros dois são: a diminuição do gasto energético e o aumento do consumo calórico. Outro fator do aumento da obesidade são as causas ambientais. Que se refere ao aumento do número de pessoas em uma família, a classe social, a falta de apoio dos pais e outros que podem atuar bastante nos hábitos alimentares e na AF (Oliveira, 2008).

\subsubsection{Obesidade infantil}

Nos últimos anos, teve um crescimento no número de crianças e adolescentes obesos, sendo que a maior parte foi por falta de $\mathrm{AF}$, com o passar dos tempos, quando essas crianças se tornam adolescentes e jovens adultos, as condições de $\mathrm{AF}$ tem uma grande diminuição. A ausência de AF e a obesidade quando seguem juntas até a vida adulta, podem ocasionar vários tipos de problemas como cardiovascular, diabetes entre outros (Juca, 2013).

Através do período de infância no início da adolescência as células adiposas crescem na quantidade e em número. Em pessoas adultas, a quantidade de células não tende a aumentar apenas no tamanho. A diminuição do peso acontece com a redução das células adiposas e não em quantidade. Para a prevenção da obesidade seria necessário impedir o progresso inicial das células adiposas, entre o tempo de crescimento (Juca, 2013). No período de infância é capaz de causar um aumento da quantidade de células adiposas, provocando um aumento exagerado de peso e acúmulo de gordura. Assim contribuindo para que as consequências sigam para vida adulta (Juca, 2013).

Tendo como alerta a obesidade infantil sendo um fator preocupante por conta de essas crianças chegarem à vida adulta obesas. De acordo com o Conselho Latino-Americano em obesidade, as crianças têm maiores chances de se transformarem em adultos obesos, sendo que cerca de $40 \%$ dessas crianças chegam a ser adolescentes obesos e dos adolescentes obesos $80 \%$ chegam à vida adulta obesa (Juca, 2013). A fim de encarar as causas relacionadas à obesidade, precisamos verificar as mudanças da gordura corporal através das etapas da vida, com objetivo de monitorar a eficácia da dieta e dos exercícios na prevenção da obesidade infantil (Juca, 2013).

\subsubsection{Obesidade na adolescência}

A obesidade na fase da adolescência e relacionada ao surgimento de problemas cardiovasculares, diabetes Melitos tipo 2, doenças psicológicas e ainda sendo prejudicial para a postura, provocando mudanças no aparelho psicomotor e ocasionando dificuldades socioeconômicas na fase adulta (Camarneiro, 2011). Uma pesquisa aponta que adolescentes com IMC com percentual maior ou igual a 75 têm maiores chances de desenvolver hipertensão arterial na fase adulta, quando igualados adolescentes com uma dieta equilibrada. Os adolescentes estando com peso elevado, especialmente do sexo masculino, têm maior possibilidade de mostrar o nível alto de colesterol ao se torna adulto (Camarneiro, 2011). 
Uma pesquisa do National Heallth and Nutrition Examination Survey (NHANESIH), feita por volta de 1976 a 1980 , mostra que o número de crianças e adolescentes acima do peso tem crescido em média 5\% dos jovens, são apontadas como risco para obesidade estabelecida pelo percentual de 95 do IMC, e 15\% são apontadas como risco para obesidade estabelecida pelo percentual de 85 do IMC (Camarneiro, 2011). Em uma pesquisa nacional de saúde e nutrição feita no Brasil, realizada no ano de 1989, apresentou que 54,7\% dos adolescentes mostraram estar no peso apropriado para sua altura, é $26,3 \%$ estavam com o peso adequado e $19 \%$ apresentava estar acima do peso adequado para sua altura. Assim apresentando alteração na descrição de doenças vem sendo analisadas no país (Camarneiro, 2011).

Dessa forma, estudos têm mostrado causas opostas para origem e a alteração da obesidade em pessoas, além disso, autores mostram que a maioria dos casos de obesidade não é apenas fatores genéticos e sim fatores socioambientais e diminuição de AF, causando o aumento exagerado de gordura corporal (Camarneiro, 2011). A medida da cintura e um excelente parâmetro para apontar a gordura visceral, com mais eficiência que o IMC, para mostrar a obesidade abdominal podendo ajudar na verificação de pessoas com maiores riscos a doenças cardiovasculares (Camarneiro, 2011).

As crianças e adolescentes obesos que dão continuidade ao acúmulo de gordura ao longo de toda a vida aumentam as chances de adquirir na vida adulta doenças cardiovasculares, respiratórias, endocrinológicas, ortopédicas, dermatológicas e psicossociais (Camarneiro, 2011).

\subsubsection{Obesidade na vida adulta}

A obesidade é destacada como uma das DANTs com diversas causas, como: fatores genéticos, metabólicos, ambientais, sociais, culturais, econômicos, estilo de vida ou ainda pode estar associada a fatores demográficos. A sua ocorrência está tanto em países desenvolvidos quanto em países em desenvolvimento. Tendo um como parâmetro de avaliação para adultos o IMC, um ferramenta capaz de proporcionar dados sobre o peso ideal de cada pessoa, sendo realizado através do peso em quilogramas $(\mathrm{kg})$ e da estatura ao quadrado $\left(\mathrm{m}^{2}\right)$. Portanto, os indivíduos que apresentam $\mathrm{IMC} \geq 30 \mathrm{~kg} / \mathrm{m}^{2} \mathrm{são}$ classificadas como obesos (Souza et al., 2018).

Para ratificar, a obesidade é definida pela OMS, como o exagerado acúmulo de gordura corporal que pode ocasionar problemas de saúde ao indivíduo. Assim, a ingestão de alimentos com um alto valor calórico e ausência de AF, podendo gerar um ganho calórico e diminuição do gasto de energia durante o dia proporcionando um acúmulo de gordura (Souza et al., 2018).

\subsubsection{Doenças relacionadas à obesidade}

Estudos mostram que o sobrepeso e obesidade estão ligados ao progresso de doenças, como: diabetes melitos tipo 2, dislipidemia, doenças cardiovasculares, doenças do aparelho respiratório, doenças do aparelho locomotor e doenças da hipertensão arterial (Sousa et al., 2018; Gaban, 2013).

\subsubsection{Diabetes Melitos Tipo 2 (DM2)}

O DM2 é um dos problemas de saúde que está mais ligada ao aumento de peso. Segundo estudos que apontam que o excesso de peso alto pode ter maiores riscos de adquirir a DM2 tanto no sexo masculino e no feminino, que o acúmulo de gordura são motivos significativo para sua evolução (Gaban, 2013). De acordo com um estudo feito em 12 capítulos brasileiros, que analisou 2.519 pessoas com diabetes tipo 2 acima de 30 anos, que a avaliação antropométrica resultou em $75 \%$ dessas pessoas estavam com sobrepeso e obesos (Gaban, 2013). 


\subsubsection{Dislipidemia}

Essa doença e frequente em pessoas que tem um maior acúmulo de gordura na parte superior do corpo, com o aumento dos triglicerídeos e da lipoproteína de maior densidade (HDL) (Gaban, 2013).

\subsubsection{Doença cardiovascular}

Pessoa com acúmulo de gordura corporal tem maiores chances de ter problema cardiovascular, como infarto do miocárdio, angina, insuficiência cardíaca digestiva, mio cardiopatia, acidente vascular cerebral (AVC), fibrilação atrial, insuficiência venosa, trombose venosa profunda e tromboembolismo pulmonar. $\mathrm{O}$ aumento do tecido adiposo pode causar um processo inflamatório de citosinas gerando uma condição inflamatória crônica, através da liberação das proteínas prótrombóticas dessa forma sendo mais contínuo em pessoas com obesidade (Gaban, 2013).

\subsubsection{Doenças do aparelho respiratório}

A apneia obstrutiva do sono é o que causa problemas respiratórios e é bastante comum em pessoas com obesidade. Sendo principal motivo de risco para o surgimento da apneia obstrutiva do sono a obesidade, atinge indivíduos do sexo masculino e feminino, com maior intensidade nos homens (Gaban, 2013). Uma pessoa obesa pode acontecer casos durante o sono como a hipopneia que é a obstrução limitada ou a apneia que é a obstrução completa. A apneia e a hipopneias são destacadas com baixa (5 a 14,9), Moderada (15 a 29), e alta maior que 30, podendo gerar riscos à saúde (Gaban, 2013).

\subsubsection{Doenças do aparelho locomotor}

Pessoas com sobrepeso ou obesas podem ter problemas nas articulações de membros inferiores como: Quadril, Joelho e Tornozelo, que são ocasionados pela osteoartrite, assim prejudicando na locomoção por apresentar fortes dores articulares (Gaban, 2013).

\subsubsection{Doença da hipertensão arterial}

A hipertensão arterial é causada deis vezes mais em obesos do que em pessoas normais, também estando relacionada a fatores genéticos, familiares e ambientais atingindo pessoas na faixa etária de 20 a 45 anos. Quando um indivíduo tem ganhado $10 \%$ a mais de gordura corporal reproduz um aumento expressivo na pressão arterial (Mariath et al., 2007).

\subsection{Atividade física}

\subsubsection{Conceito}

Com o intuito de prevenir alguns riscos de doenças e na promoção da saúde podendo destacar a prática regular de AF como fator importante. No entanto, fazendo uma diferenciação entre AF e exercício. Segundo Caspersen, Powell e Christenson (1985), o termo AF está relacionado a qualquer movimento que corpo realiza através do sistema muscular esquelético, que resulta em um maior gasto energético se comparado à taxa metabólica em repouso. No entanto, o EF está inserido no tema AF, por ser uma atividade de caráter planejado, repetitivo e intencional, com objetivo de manter, melhorar um ou mais componentes da aptidão física (Silva \& Costa, 2011).

No momento atual o exercício físico (EF) é visto como algo sincrônico de desempenho que liga total movimentação do corpo, porém, tem em si uma definição diferenciada por motivo do quadro em que é executado. Apresentando como centro indispensável à prática de avaliação nos níveis de EF se torna importante estar relacionado na conscientização e nos demais temas que tem conexão com a AF e o gasto energético (Moreira, 2014).

Desta forma, compreende-se que há diversos princípios para o EF, preferindo-se o conceito de Carpensen et al. (1985) 
onde o EF é visto do modo que total e qualquer movimentação do corpo são realizados pelos músculos esqueléticos que gera um desgaste energético elevado das condições de descanso. Com base neste conceito, é viável compreender, no entanto, que todo movimento realizado pela musculatura do corpo tem efeito de gasto intenso nos exercícios executados e realizados por uma pessoa em sua rotina diária. É comum, o EF ser visto na realização no qual se apresenta as tarefas em que os indivíduos se locomovem diariamente, nas tarefas do lar ligadas as atividades de casa, nos exercícios de distração que é realizado em horas vagas, nas horas de EF, no trabalho é, na situação dos jovens, no colégio (Moreira, 2014).

\subsubsection{Benefícios da atividade física para a saúde}

Recentemente, a ausência de AF vem crescendo cada vez, mas sendo um dos primeiros causadores de risco para a evolução de muitas as doenças crônicas, realidade que vem se tornando um grande problema na saúde das pessoas (Ceschini, 2007). Vários procedimentos publicados durante os últimos tempos têm relatado de maneira direta e de fácil entendimento os riscos que as pessoas passam com a inatividade física e que ela pode provocar para a saúde, principalmente, quando relacionada a diversas riscos, como o uso de cigarro, dieta de forma incorreta, taxas altas de colesterol sanguíneo, pressão arterial alta, peso elevado e obesidade (Ceschine, 2007).

Porém os estudos têm mostrado de maneira extremamente clara a relação que existe tendo uma prática frequente de AF e suas vantagens na saúde das pessoas independente de sua idade, cultura, nível de vida (Ceschini, 2007). A maneira de se viver em um padrão de vida de modo ativo com a prática de EF, tem várias vantagens na saúde das pessoas sendo também importante no cuidado, domínio, tratamento ou correção (Matsudo \& Matsudo, 2001). Com o desempenho frequente e habitual de AF, temos resultados positivos e satisfatórios sendo (Ceschine, 2007):

- Benefícios biológicos ou fisiológicos: redução do peso do corpo, redução da pressão arterial, melhoria da forma lipídica, domínio da glicose, melhorias na respiração, evolução da força e vigor muscular (Ceschine, 2007);

- Benefícios psicológicos: vantagens no bem-estar, diminuição da depressão, mau humor e entre outros (Ceschine, 2007);

- Benefícios cognitivos: melhorias na questão de paciência, atenção, concentração e entre outros (Ceschine, 2007).

Na presença das vantagens e privilégios com o desempenho habitual de praticar AF onde se tem um ótimo benefício para a saúde, já vislumbrada na literatura, sendo nacional e internacional onde tem sido um grande aliado para combater as doenças crônicas não transmissíveis e reduzir as taxas de mortalidade, além de estar relacionado nas melhores condições de vida (Ceschini, 2007).

\subsubsection{Dados sobre a prática da atividade física}

Seguindo referências internacionais, qualificou-se que a realização da $\mathrm{AF}$ em programas de lazer são suficiente no mínimo 30 min. diários de AF de intensidade leve ou moderada com a frequência de 5 dias semanais ou mais; ou a prática de no mínimo 20 min. por dia de AF de intensidade vigorosa em 3 dias semanais ou mais. São apontadas como práticas de intensidade leve ou moderada: caminhada, caminhada em esteira, musculação, hidroginástica, ginástica em geral, natação, artes marciais, ciclismo e voleibol e as seguintes práticas a pontadas como intensidade vigorosa; corrida, corrida em esteira, ginástica aeróbica, futebol, basquetebol e tênis.

No entanto, destaca-se como situação de sedentarismo as pessoas que confirmaram: (i) não realizar qualquer AF no lazer nos três últimos meses; (ii) não ocorrer esforços físicos intensos no trabalho (não andar muito, não carregar peso e não desenvolver outras atividades semelhantes, em situações de esforço físico); (iii) não se locomover para o trabalho a pé ou de bicicleta; e (iv) não ser em carregado dos atividades domésticas pesados de suas residências (Malta et al., 2006).

Segundo a OMS (2004) determina que a realização de AF, segue como ideal para adultos uma prática moderada de 
pelo menos 150 min. semanais ou uma prática de AF vigorosa de 75 min. semanal, que podem ser divididos no mínimo em 10 min. de duração, sem definição de frequência por semana. Por sua vez um estudo realizado na cidade de Minas Gerais, à AF foi indicada do seguinte modo: sendo a prática ideal de no mínimo de $150 \mathrm{~min}$. por semana; e de formar intermediária a realização de 1 a 149 min. por semana; e considerada ruim quando não há uma prática de AF moderada ou vigorosa (Dias et al., 2017).

Informações mostram que $17 \%$ da população mundial é classificada como não praticante de $\mathrm{AF}$ e $60 \%$ não correspondem ao mínimo de AF recomendável. A população mundial é afetada por volta de 70\% pelo sedentarismo, sendo a pontado pela OMS por fator determinante na causa de dois milhões de mortes no mundo inteiro e responsável por $75 \%$ dos óbitos nas Américas (Dias et al., 2017). A ponta que o estilo de vida atual tem como fatores indispensáveis que estão relacionados à falta de $\mathrm{AF}$ os seguintes: o trabalho usa de transportes motorizados e atividades de lazer relacionados às tecnologias como televisão, computadores e vídeo games (Dias et al., 2017).

\subsubsection{Treinamento Aeróbio}

Em conformidade com Glaner (2003), treinamento aeróbico (TA) relaciona-se à função cardiovascular e pulmonar e podem ser descritas pela resistência aeróbica elemento da aptidão física associada à saúde. De acordo com Brum, Forjaz, Tinucci e Negrão (2004) explica que a resistência aeróbia é classificada como atividades que promovem a diminuição dos riscos de problemas cardiovasculares, isto é, trazendo mais benefícios para o sistema circulatório. Sendo assim, destacam-se como exercícios que envolvem grandes grupos musculares podendo ser classificados: dança, hidroginástica, caminhada, corrida, natação, ciclismo, entre outros (Lima et al., 2014).

No entanto, para que a AF proporcione benefícios, é essencial analisar, pelo menos três aspectos: frequência, intensidade e duração do exercício (Lima et al., 2014). De acordo com Lima, Levy e Luiz (2014), informaram que a prática de treinamento aeróbico com apenas um dia por não são eficientes para proporcionar em curto prazo uma melhora na qualidade de vida. Contudo, se realizada no mínimo 2x/semana, os aspectos vitaliciedade, função cardiovascular, percepção de dor corporal e saúde mental desenvolvem expressivamente (Lima et al., 2014). Por sua vez, o TA com uma intensidade moderada, por pelo menos 30 min., três a cinco dias por semana, pode proporcionar efeitos benéficos para saúde cardiovascular (Lima et al., 2014).

\subsubsection{Treinamento anaeróbio ou de força}

De acordo com Geraldes (2003), o TF podem ser descritos de várias formas como: treinamento contra a resistência, musculação ou treinamento resistido são definições usadas para representar as diversas classificações do treinamento de força, resistência ou potência muscular e eventualidade de fisiculturismo ou levantamentos de peso. O TF não e descrito apenas por levantamento de pesos, assim como, a utilização de maquinas como meio de resistência ou de faixas elásticas. Teoricamente, o treinamento com pesos retrata-se ao levantamento dos pesos livres e pelas máquinas que ocasionam a realização de resistência dinâmica invariável e variável (Farias \& Rodrigues, 2009).

Segundo Geraldes (2003), o tema denominado de princípios e variáveis metodológicas do TF. O TF vem sendo utilizado com uma das formas mais populares de exercícios para conseguir diversos pontos relacionados à aptidão física, que resulta em efetividade importante a continuidade da saúde, diminui o processo de envelhecimento e diminui os índices de enfermidades ocasionadas pelo sedentarismo. Com finalidade de que o programa de treinamento físico visando aptidão física seja visto como completo, é importante a inclusão do TF e evidência as características associadas a saúde e desempenho atlético: composição corporal, resistência cardiovascular, força muscular, resistência muscular, flexibilidade, agilidade, equilíbrio, potência, coordenação motora e tempo de reação (Farias \& Rodrigues, 2009). 
Um programa de TF, elaborado e estruturado pode apresentar melhoras importantes na força e hipertrofia muscular, na densidade óssea e na flexibilidade (Farias \& Rodrigues, 2009). Embora, a musculação não utiliza como fonte principal o tecido adiposo no momento do exercício e dispõe do sistema mais intenso as vias energéticas ATP-PC e glicólise anaeróbia, ocorrem um alto emprego de lipídios entre uma série e outra dos exercícios, por conta dos treinamentos aeróbicos aumentado, com objetivo de restabelecer os sistemas anaeróbios depletados. Além disso, o metabolismo permance elevado por muitas horas, o que aumenta a oxidação de gordura (Farias \& Rodrigues, 2009).

\subsection{Atividade física e obesidade}

\subsubsection{Treinamento aeróbio e obesidade}

O TA apresenta-se como uma forma muito usada em programas de emagrecimento em meio de EF sendo mais eficiente para redução de massa corporal, particularmente no tecido adiposo. Isto se acontece pela elevação do déficit energético proporcionado pelo EF, tanto durante como depois do término dele. Porém, o aumento do consumo de oxigênio mesmo após o término do esforço é uma das causas positivas do exercício aeróbio em redução à perda e/ou manutenção do peso corporal (Dias et al., 2014).

São considerados TA, tais como: caminhada, corrida em ritmo moderado (jogging), natação, ciclismo, jogos coletivos (basquetebol, voleibol, futebol etc.) e modalidades praticadas individualmente, como por exemplo, esportes com raquetes. Esses devem ter uma intensidade moderada (dentro da zona alvo da frequência cardíaca), a necessidade de energia deve ser gradualmente mais alta até chegar cerca de 2000 a 2500 Kcal por semana, com o comprometimento dos grandes grupos musculares, de forma dinâmica e num ritmo moderadamente progressivo (Salve, 2006).

O TA pode ser classificado em dois métodos: contínuo, que é capaz de variar a intensidade por volta de 50 a $85 \%$ do $\mathrm{VO}_{2 \text { máx }}$, e pode ter uma duração de 30 a 60 min. e intervalado, não se permanece em "steady state" contínuos. Toda via, concentram-se nas elevadas porcentagens de $\mathrm{VO}_{2 \text { máx }}$ Esta é uma ferramenta importante, pois acarreta adequações relevantes no transporte de $\mathrm{O}_{2}$ e nos tecidos, pois decorrem melhoras da capacidade de suportar e metabolizar concentrações altas de ácido láctico (Salve, 2006).

De acordo Shangold (1990), menciona que o TA com aumento da frequência cardíaca e da oxigenação tecidual proporciona redução expressiva do tecido adiposo da região abdominal em homens, se comparado a mulheres. Porém, o EF primeiramente contribui para aumento da massa muscular e redução de massa gorda, não acontecendo redução de peso corporal. Só depois de três ou quatro meses ocorrera reduções consideráveis do tecido adiposo, ocasionando a redução de peso (Salve, 2006).

O TA é capaz de auxiliar no emagrecimento sendo usado com uma intensidade de moderada a alta $\left(70 \%\right.$ do $\left.\mathrm{VO}_{2 \mathrm{máx}}\right)$, assim, aumenta a taxa metabólica de repouso depois do exercício por volta de 5 e 16\%, sendo capaz de continuar elevada por 12 a 39 horas (Pontes, Sousa \& Navarro, 2009). No entanto no exercício aeróbio a oxidação da gordura é muito mais alta se comparada ao treinamento com pesos (Pontes et al., 2009).

\subsubsection{Treinamento Anaeróbio e Obesidade}

A musculação (exercício contra resistência) se expressa através de contrações musculares de maneira repetitiva de encontro a uma resistência, e tem vários objetivos, entre eles o emagrecimento (Schurt; Liberali \& Navarro, 2016). O TF vem sendo usado como um dos recursos para o tratamento da obesidade tendo uma conduta auxiliar (ACSM, 2002), pois o gasto calórico proporcionado pelo EF se apresenta como importante fator na perda de gordura corporal, e na melhora dos níveis de aptidão física (Neves, Martins, Souza, \& Júnior, 2015). 
O TF vem sendo destacado como uma forma surpreendente para melhorar a qualidade de vida, além de contribuir para o tratamento de várias doenças (Graves \& Franklin, 2006). Contudo, alguns estudos analisaram unicamente os efeitos desse método de treinamento no tratamento da obesidade (Hannibal et al., 2010). Os programas de TF proporcionam meios de controlar o peso corporal melhorando a capacidade de gastar energia, a massa muscular e a taxa metabólica de repouso, além do gasto de oxigênio depois do exercício. Conforme os efeitos, os percentuais de gordura vão reduzindo, contribuindo para o emagrecimento (Hannibal et al., 2010).

Ainda, a importância no TF para aumentar a força e resistência muscular pode trazer benefícios para as atividades do dia a dia, proporcionando adesão de um estilo de vida com um maior nível de atividade em pessoas obesas sedentárias (Hannibal et al., 2010). Santos e Romanholo (2008) analisaram as finalidades da musculação a respeito da redução do tecido adiposo e a modulação no metabolismo basal. Através de um estudo com a participação de 27 mulheres com uma média de idade de 27,7 anos, que foi realizado no período de 8 semanas de treinamento (Schurt, Liberali \& Navarro, 2016).

\section{Resultados e discussão}

O Quadro 1 abaixo apresentam os 5 estudos dos 25 artigos para sintetizar a leitura e visibilidade dos resultados aos benéficos da atividade físicas em indivíduos com excesso de peso corporal. Desta forma, a recente pesquisa através do método de revisão bibliográfica teve por objetivo verificar os efeitos da AF em indivíduos obesos.

Quadro 1. Apresentação dos estudos sobre os efeitos benéficos da atividade física em indivíduos obesos.

\begin{tabular}{|c|c|c|c|}
\hline Autores & $\begin{array}{c}\text { Benefícios gerais da } \\
\text { atividade física }\end{array}$ & $\begin{array}{c}\text { Benefícios do treinamento } \\
\text { aeróbico }\end{array}$ & Benefícios do treinamento de força \\
\hline Ceschini, 2007. & $\begin{array}{c}\text { Biológicos ou fisiológicos: } \\
\text { redução do peso do corpo, } \\
\text { redução da pressão arterial, } \\
\text { melhoria da forma lipídica, } \\
\text { domínio da glicose, } \\
\text { melhorias na respiração, } \\
\text { evolução da força e vigor } \\
\text { muscular. } \\
\text { Psicológicos: vantagens no } \\
\text { bem-estar, diminuição da } \\
\text { depressão, mau humor e } \\
\text { entre outros. } \\
\text { Cognitivos: melhorias na } \\
\text { questão de paciência, } \\
\text { atenção, concentração e } \\
\text { entre outros. }\end{array}$ & & \\
\hline Pontes et al., 2009. & & $\begin{array}{l}\text { O TA é capaz de auxiliar no } \\
\text { emagrecimento sendo usado } \\
\text { com uma intensidade de } \\
\text { moderada a alta ( } 70 \% \\
\text { VO2máx), assim, aumenta a } \\
\text { taxa metabólica de repouso } \\
\text { depois do exercício por volta de } \\
5 \text { e } 16 \% \text {, sendo capaz de } \\
\text { continuar elevada por } 12 \text { a } 39 \\
\text { horas. }\end{array}$ & \\
\hline Dias et al., 2014. & & $\begin{array}{l}\text { O TA apresenta-se como uma } \\
\text { forma muito usada em } \\
\text { programas de emagrecimento em } \\
\text { meio de EF sendo mais eficiente } \\
\text { para redução de massa corporal, } \\
\text { particularmente no tecido } \\
\text { adiposo. Isto se acontece pela }\end{array}$ & \\
\hline
\end{tabular}




\begin{tabular}{|c|c|c|}
\hline & $\begin{array}{l}\text { elevação do déficit energético } \\
\text { proporcionado pelo EF, tanto } \\
\text { durante como depois do término } \\
\text { dele. Porém, o aumento do } \\
\text { consumo de oxigênio mesmo } \\
\text { após o término do esforço é uma } \\
\text { das causas positivas do } \\
\text { treinamento aeróbico em } \\
\text { redução à perda e/ou } \\
\text { manutenção do peso corporal. }\end{array}$ & \\
\hline Hannibal et al., 2010. & & $\begin{array}{l}\text { TF proporcionam meios de controlar o peso } \\
\text { corporal melhorando a capacidade de gastar } \\
\text { energia, a massa muscular e a taxa metabólica de } \\
\text { repouso, além do gasto de oxigênio depois do } \\
\text { exercício. Conforme os efeitos, os percentuais de } \\
\text { gordura vão baixando, contribuindo para o } \\
\text { emagrecimento. Que consistiu em } 5 \text { exercícios } \\
\text { com } 3 \text { series em cada exercício com } 8 \text { repetições } \\
\text { nas } 2 \text { primeiras series a } 80 \% \text { da repetição máxima } \\
\text { (1RM). E na última série foi realizado quantas } \\
\text { repetições conseguissem até } 12 \text { repetiçôes. }\end{array}$ \\
\hline Schurt et al., 2016. & & $\begin{array}{l}\text { TF diminui o tecido adiposo e modula o } \\
\text { metabolismo basal. Através de um estudo com a } \\
\text { participação de } 27 \text { mulheres com uma média de } \\
\text { idade de } 27,7 \text { anos, que foi realizado num periodo } \\
\text { de } 8 \text { semanas de treinamento. }\end{array}$ \\
\hline
\end{tabular}

Fonte: Autores (2019).

Por meio de resultados foi capaz de compreender que AF de forma geral promove benefícios para indivíduos hipertensos e não hipertensos. Segundo Ceschine (2007), AF proporciona benefícios fisiológicos apresenta a melhora na redução de peso corporal. Já em relação ao treinamento aeróbico, Pontes et al. (2009), mostram que é capaz de auxiliar no emagrecimento sendo usado com uma intensidade de moderada a alta $\left(70 \% \mathrm{VO}_{2}\right.$ máx). Já o estudo apresentado por Dias et al. (2014), apresentaram que a redução da gordura corporal acontece pela elevação do déficit energético proporcionado pelo exercício, tanto durante como depois do término dele. Em relação ao treinamento de força Hannibal et al. (2010), apresentam que os treinamentos com pesos proporcionam meios de controlar o peso corporal melhorando a capacidade de gastar energia, a massa muscular e a taxa metabólica de repouso, além do melhor gasto de oxigênio depois do exercício.

No primeiro estudo que menciona AF de forma geral citada por Ceschine (2007), mostra que proporciona benefícios fisiológicos, psicológicos e cognitivos, sendo que nos efeitos benefícos fisiologicos apresenta a melhora na redução de peso por meio da prática de AF (Ceschine, 2007). Já em relação ao TA o estudo realizada por Pontes et al. (2009), mostra que é capaz de auxiliar no emagrecimento sendo usado com uma intensidade de moderada a alta $\left(70 \% \mathrm{VO}_{2 m a ́ x}\right)$, assim, aumenta a taxa metabólica de repouso depois do exercício por volta de 5 e 16\%, sendo capaz de continuar elevada por 12 a 39 horas (Pontes et al., 2009). Adicionalmente, em outro estudo relacionado ao TA mencionado por Dias et al. (2014), indicou que se apresenta como uma forma muito utilizada em programas de emagrecimento em meio de EF sendo mais eficiente para redução de massa corporal, particularmente no tecido adiposo. Isto acontece pela elevação do déficit energético proporcionado pelo exercício, tanto durante como depois do término dele. Porém, o aumento do consumo de oxigênio mesmo após o término do esforço é uma das causas positivas do TA em redução à perda e/ou manutenção do peso corporal. Ambos os estudos relacionados ao TA mostraram melhoras na redução de peso corporal (Dias et al., 2014).

Os resultados apresentados por dois estudo voltados ao treinamento resistido sendo que o primeiro mencionado por Hannibal et al. (2010), apresentando que o treinamento com pesos proporciona meios de controlar o peso corporal melhorando a capacidade de gastar energia, a massa muscular e a taxa metabólica de repouso, além do melhor gasto de oxigênio depois do exercício. Conforme os efeitos, os percentuais de gordura vão reduzindo, contribuindo para o emagrecimento. Que consistiu 
em 5 exercícios com 3 séries em cada exercício com 8 repetições nas 2 primeiras series a 80\% da 1RM. E na última série foi realizado 12 repetições até o máximo (Hannibal et al., 2010). No segundo estudo mencionado por Schurt et al. (2016), mostraram que o treinamento resistido diminuiu do tecido adiposo e modulou o metabolismo basal. De acordo com os achados de Schurt et al. (2016) apresentaram que mulheres com média de idade de 27,7 anos obtiveram redução no percentual de gordura ao serem submetidas ao treinamento resistido no período de 8 semanas.

Sendo assim, os estudos apresentados no presente estudo observamos que a prática de atividade física regular promove a melhora na redução de gordura corporal, seja ela atividade física de forma geral ou através dos treinamentos: aeróbico ou de força por meio de exercícios como: caminhada, corrida e ciclismo, treinamento com pesos entre outros.

\section{Conclusão}

Portanto, a pesquisa realizada por meio do método de revisão bibliográfica sobre AF e obesidade concluiu que a maioria dos artigos e monografias que foram analisados apresentaram benefícios da AF em indivíduos obesos. Com isso, é importante destacar que a prática de tais atividades que envolvem maiores grupos musculares, como a caminhada, corrida e ciclismo e treinamento com pesos podem proporcionar reduções na gordura corporal. Pois a literatura apresenta que além da AF auxiliar na redução de gordura corporal também ajuda a diminuir os riscos de doenças associadas a patologia.

Desta forma, tanto o TA quanto o de TF mostraram resultados importantes na redução de gordura corporal proporcionando melhora na qualidade de vida. Além disso, os TA e de TF contribuem para prevenção e tratamento da obesidade. No entanto, é muito importante alertar a população sobre o que é obesidade e quais a suas causas e doenças relacionadas a patologia. Por fim, a prática regular de AF proporciona melhoras na redução de gordura corporal e atenua a progressão de doenças evitando-se assim a morbidade e a mortalidade.

Sendo assim, o tema obesidade e exercício físico é um conteúdo bastante complexo, e com isso, são necessárias mais pesquisas sobre a relação dessas duas vertentes. Portanto, almejamos realizarmos trabalhos futuros sejam originais sejam revisões integrativas e narrativas baseadas em vias de sinalização celular para determinar os comportamentos e formas de proteínas responsáveis pelo metabolismo e respostas fisiopatológicas.

\section{Referências}

Almeida, S. S. Nascimento, P. C. B. D. \& Quaioti, T. C. B. (2002). Quantidade e qualidade de produtos alimentícios anunciados na televisão brasileira. Revista de Saúde Pública, 36 (3), 353-355.

American College Sports Medicine (2002). Progression models in resistance training for healthy adults. Medicine Science Sports Exercise, 34(2), 364-80.

Anjos, L. A. Teixeira, F. C. Wahrlich, V. Vasconcellos, M. T. L. \& Going, S. B. (2013). Percentage body fat and body mass index in a urban Brazilian adult probability sample. Cadernos Saúde Pública, 29 (1), 73-81.

Brasil, Ministério da Saúde. Vigitel — Brasil 2018 (2019): Vigilância de Fatores de Risco e Proteção para Doenças Crônicas por Inquérito Telefônico. Brasília, DF: Ministério da Saúde., Recuperado de https://portalarquivos2.saude.gov.br/images/pdf/2019/julho/25/vigitel-brasil-2018.pdf

Brum, P. C., Forjaz, C. L. M., TinuccI, T. \& Negrão, C. E. (2004). Adaptações agudas e crônicas do exercício físico no sistema cardiovascular. Revista Paulista de Educação Física, 18, 21-31.

Camarneiro, J. M. (2011). Análise da composição corporal em adolescentes obesos (Tese de Doutorado) Universidade Estadual Paulista, “Júlio De Mesquita Filho", Faculdade de Ciências Farmacêuticas, Araraquara, São Paulo.

Caspersen, C. J.., Powell, K. E., \& Christensen, G. M. (1985). Physical activity, exercise, and physical fitness: definitions and distinctions for health-related research. Public Health Reports, 100(2), 126-131.

Ceschini, F. L. (2007). Nível de atividade física em adolescentes de uma escola do dristrito da Vila Nova Cachoeirinha em São Paulo-SP (Dissertação de Mestrado).Universidade de São Paulo, São Paulo.

Dias, I. B., Montenegro, R. A., \& Monteiro, W. D. (2014). Exercícios físicos como estratégia de prevenção e tratamento da obesidade: aspectos fisiológicos e metodológicos. Revista HUPE, 13(1), 70-79. 
Dias, J., Junior, M. D., Costa, M. A. R., Francisqueti, V., \& Higarashi, I. H. (2017). Prática de atividade física em docentes do ensino superior: foco na qualidade de vida. Escola Anna Nery, 21 (4), 1-6.

Farias, I. G., \& Rodrigues, T. S. (2009). Exercício resistido: Na saúde, na doença e no envelhecimento (Trabalho de Conclusão de Curso). Universidade Estadual Paulista “Júlio de Mesquita Filho”, Lins, São Paulo.

Glaner, M. F. (2003). Importância da aptidão física relacionada à saúde. Revista Brasileira de Cineantropometria \& Desempenho Humano, 5 (2), 75-85.

Gaban, S. C. (2013). Excesso de peso e obesidade central em população adulta urbana-fatores associados e doenças relacionadas ao risco cardiovascular (Dissertação de Mestrado). Universidade Federal do Mato Grosso do Sul, Campo Grande.

Graves, J. E. \& Franklin, B. A. (2006). Treinamento Resistido na Saúde e Reabilitação. Revinter.

GeraldeS, A. A. R. (2003). Exercício como estratégia de prevenção e tratamento da osteoporose: Potencial e limitações. Revista Brasisileira de Fisiologia do Exercício, 2 (1), 1-28.

Hannibal, D., Zolet, N. E., Souza, J. C. de, Speretta, G. F. F., Leite, R. D., \& Prestes, J. (2011). Exercício físico e obesidade: o impacto das diferentes modalidades. Revista Brasileira de Prescrição e Fisiologia do Exercício, 4(20), 218-229.

Instituto Brasileiro de Geografia e Estatística (2015). Pesquisa nacional de saúde: 2013, ciclos de vida, Brasil e grandes regiões - Coordenação de Trabalho e Rendimento. IBGE. https://biblioteca.ibge.gov.br/visualizacao/livros/liv94522.pdf.

Instituto Brasileiro de Geografia e Estatística (2010). POF 2008-2009: desnutrição cai e peso das crianças brasileiras ultrapassa padrão internacional. IBGE. https://censo2010.ibge.gov.br/noticias-censo?busca=1\&id=1\&idnoticia=1699\&t=pof-20082009-desnutricao-cai-peso-criancas-brasileiras-ultrapassa-padraointernacional\&view=noticia

Jucá, E. O. (2013). Análise da correlação dos índices de adiposidade corporal, massacorporal e do percentual de gordura corporal em escolares do municipio de Porto Velho (Trabalho de Conclusão de Curso). Universidade Federal de Rondônia, Porto velho.

Lima, D. F., Levy, R. B. \& Luiz, O. C. (2014). Recomendações para atividade física e saúde: consensos, controvérsias e ambiguidades. Revista Panamericana Salud Pública, 36 (3), 164-70.

Lima, R. P. (2012). Sobrepeso, obesidade e IMC ajustado por diversos fatores na população de um município Brasileiro em todas sa faixas etárias (Dissertação de Mestrado). Universidade Federal da Paraíba, João pessoa.

Matsudo, S. M.., Matsudo, V. K. R.., \& Barros Neto, T. L. (2001). Atividade física e envelhecimento: aspectos epidemiológicos. Revista Brasileira de Medicina do Esporte, 7(1), 2-13.

Malta, L. C., Moura, E. C., Castro, A. M., Cruz, D. K. A., Morais Neto, O. L. \& Monteiro, C. A. (2009). Padrão de atividade física em adultos brasileiros resultados de um inquérito por entrevistas telefônicas, 2006. Epidemiologia e Serviços de Saúde, 18(1), 7-16.

Mariath, A. B., Grillo, L. P.., Silva, R. O., Schmitz, P., Campos, I. C., Medina, J. R.C. \& Kruger, R. M. (2007). Obesidade e fatores de risco para o desenvolvimento de doenças crônicas não transmissíveis entre usuários de unidade de alimentação e nutrição. Cadernos Saúde Pública, 23(4), 897-905.

Moreira, R. B. (2014). Níveis de atividade física nas aulas de educação física (Tese de Doutorado). Universidade Federal do Rio Grande do Sul, Porto Alegre, $1-101$.

Martins, G. A. (1994). Manual para elaboração de monografias e dissertações. Atlas.

Neves, D. R., Martins, E. A., Souza, M. V. C. \& Junior, A. J. S. (2015). Efeitos do treinamento de força sobre o índice de percentual de gordura corporal em adultos. Revista Brasileira de Obesidade, Nutrição e Emagrecimento, 9(52),135-141.

Oliveira, A. B. (2008). Impacto dos estratos econômicos na prevalência do sobrepeso e da obesidade em escolares de Maringá-PR Brasil (Dissertação de Mestrado). Universidade Federal de Maringá, Paraná, 1-101.

Organização Mundial da Saúde (2004). Obesidade: prevenindo e controlando a epidemia global. Roca.

Organização Mundial da Saúde (2021). Vigilância Alimentar e Nutricional: Curvas de Crescimento da Organização Mundial da Saúde. https://aps.saude.gov.br/ape/vigilanciaalimentar/curvascrescimento.

Pinheiro, A. R. O., Freitas, S. F. T., \& Corso, A. C. T. (2004). Uma abordagem epidemiológica da obesidade. Revista de Nutrição, 17(4), 523-33.

Pontes, A. L., Sousa, I. A. \& Navarro, A. C. (2009). O tratamento da obesidade através da combinação dos exercícios físicos e terapia nutricional visando o emagrecimento. Revista Brasileira de Obesidade, Nutrição e Emagrecimento, 3(14), 124 -135.

Salve, M. G. (2006). Obesidade e Peso Corporal: riscos e consequências. Movimento \& Percepção, 6 (8), 29 - 48.

Schurt, A., Liberali, R. \& Navarro, F. (2016). Exercício contra resistência e sua eficácia no tratamento da obesidade: uma revisão sistemática. Revista Brasileira de Obesidade, Nutrição e Emagrecimento, 10 (59), 215-223.

Silva, P. V. \& Costa, A. L. (2011). Efeitos da atividade física para a saúde de crianças e adolescentes. Psicologia Argumento, 29 (64), 41-50.

Silva, T. M. (2017). Obesidade geral e abdominal e seus determinantes em uma população adulta residente na zona rural do município de Pelotas, RS (Dissertação de Mestrado). Universidade Federal de Pelotas, Rio Grande do Sul, 1-178.

Souza S. A., Silva, A. B., Cavalcante, U. M. B., Lima, Caliandra, M. B. L. \& Souza, T. C. (2018). Obesidade adulta nas nações: uma análise via modelos de 
Research, Society and Development, v. 10, n. 8, e49410815286, 2021

(CC BY 4.0) | ISSN 2525-3409 | DOI: http://dx.doi.org/10.33448/rsd-v10i8.15286

regressão beta. Cadernos de Saúde Pública, 34 (8), 1-13.

Santos, C. N., \& Romanholo, R. A. (2008). Treinamento resistido de musculação para o controle de peso de mulheres entre 18 e 30 anos de idade de uma academia do município de Cacoal-RO. Revista Brasileira de Obesidade, Nutrição e Emagrecimento, 2(12), 584-587.

Shangold, M. M. (1990). Exercise in the menopausal wimon. Obstetrics and Gynecology, 75 (4), 53-58.

Tavares, T. B., Nunes, S. M. \& Santos, M. O. (2010). Obesidade e qualidade de vida: revisão da literatura. Revista de Medicina de Minas Gerais, 20(3), 359366. 